\title{
Fuzzy Modeling with Immune Multi-Objective Algorithm to Optimal Allocate of Capacitors in Distribution Systems
}

\author{
Tsong-Yau Huang ${ }^{1}$, Ying-Tung Hsiao ${ }^{2}$, Chia-Pang Chen ${ }^{3}$, and, Chih-Han Chang ${ }^{3}$ \\ ${ }^{1}$ Department of Mechanical Engineering, Chinese Culture University, Taiwan \\ ${ }^{2}$ Department of Computer Science, National Taipei University of Education, Taiwan \\ ${ }^{3}$ Department of Electrical Engineering, Tamkang University, Taiwan
}

\begin{abstract}
This work proposes a two-stage immune algorithm that embeds the compromise programming to perform multi-objective optimal capacitor placement. A new problem formulation model that involves fuzzy sets to reflect the imprecise nature of objectives and incorporates multiple planning requirements is presented. The proposed approach finds a set of noninferior (Pareto) solutions rather than any single aggregated optimal solution. Additionally, this developed approach eliminates the need for any userdefined weight factor to aggregate all objectives. Comparative studies are conducted on an actual system with encouraging results, demonstrating the effectiveness of the proposed presented approach.
\end{abstract}

Keywords: Fuzzy modeling, Capacitor placement, immune algorithm, non-inferior set, compromise programming.

\section{INTRODUCTION}

Typical distribution systems operate in a radial configuration; they are supplied from substations and feed to distribution transformers. The spatial density of the load is high in urban areas, where underground cables and large transformers are used, but lower in mixed and rural areas, where overhead lines and smaller transformer units are used. Numerous shunt capacitors are installed along distribution feeders to compensate for reactive power to regulate the voltage; reduce energy; correct the power factor, and release system capacity, for both urban and rural areas. The general capacitor placement problem is to locate and determine the sizes of capacitors to be installed at the nodes of a radial distribution system under various loading conditions.

Various attempts from different perspectives have been made to solve the capacitor placement problem. For instance, the problem has been formulated as a mixed integer programming problem in which power flows and voltage constraints are applied [1]. Optimal capacitor planning has been implemented based on the fuzzy algorithm in practical distribution systems [2]. A solution technique based on simulated annealing (SA) has been developed; implemented in a software package, and tested on a real distribution system with 69 buses $[3,4]$. The Tabu Search technique has been applied to determine the optimal capacitor planning in the distribution system used in [5], and the results of the TS compared with those of the SA. Genetic algorithms (GA) have been used to determine the optimal selection of capacitors [6].

Notably, most of these approaches treat the capacitor placement problem as a single objective problem. However, in recent years, customers have made strong demands of electrical utility companies [7]. Various problems have multiple and conflicting objectives, which make the optimization problem interesting to solve. No single solution is an optimal solution to a problem with multiple conflicting objectives, so a multi-objective optimization problem has a number of trade-off optimal solutions. Classical optimization methods can at best find one solution in one simulation run, so such methods inconvenient when used to solve multi-objective optimization problems.

In light of the above, this study formulates the capacitor placement problem as a multiple objective problem, including operational requirements. This work also presents a two-staged immune algorithm to solve the constrained multiple objective problem.

\section{Problem Formulation}

This study formulates the capacitor allocation problem to determine the locations and size of capacitors to be installed in the nodes of a radial distribution system under various loading conditions. The problem formulation considers four objective functions, to minimize the total cost of capacitors to be installed, 
the energy loss and the deviation of bus voltage, and to maximize the system security margin of transformer capacity.

\subsection{Minimizing capacitor construction expenditure}

The cost of capacitors includes the purchase cost and installment and maintenance cost.

$$
\min f_{c}=\sum_{i \in \Psi} \frac{1}{\mathrm{y}}\left[k_{p}(q)+k_{m}(q)\right] a_{i}
$$

where $a_{i}$ is a $0-1$ decision variable: $a_{i}=1$ if the ith bus is selected for capacitor installation; otherwise $a_{i}=0$;

$\Psi$ represents the set of candidate locations of buses to be considered for capacitor injection; $y$ denotes the life time (years) of the capacitors; $k_{p}$ represents the purchased cost of capacitors of capacitance $q ; k_{m}$ denotes the fixed installment and maintenance cost. Figure 1 plots the fuzzy membership function $f_{c}$ of the cost.

\subsection{Minimizing real power loss}

The total cost of the real power loss from line branches, is defined as,

$$
\min f_{e}=\sum_{j=1}^{n_{t}} k_{e j} t_{j} p_{\text {loss }, j}
$$

where $n_{t}$ represents the total number of load levels; $k_{e j}$ represents the cost of power under load $j$; $t_{j}$ represents the duration of the application of load $j$, and $p_{\text {loss }, j}$ is the total real power loss of the considered system under load $j$. Figure 2 displays the fuzzy membership function of power loss.

\subsection{Minimizing Deviation of Bus Voltage}

An index is defined that quantifies the deficiency in the system caused by the bus voltage.

$$
\min f_{v}=\max _{i}\left|v_{i}-v_{i}^{\text {Rated }}\right|, i=1,2,3, \ldots, n_{b}
$$

where $n_{b}$ is the total number of buses; $v_{i}$ and $v_{i}^{\text {Rated }}$ denote the real and rated voltages of bus $i$, respectively, and $f_{v}$ represents the maximal deviation of the bus voltage in the system. A lower $f_{v}$ corresponds to a higher quality voltage profile and better system security. Figure 3 plots the fuzzy membership function of the deviation of the bus voltage where $f_{v \max }$ is the maximum allowable deviation of bus voltage.

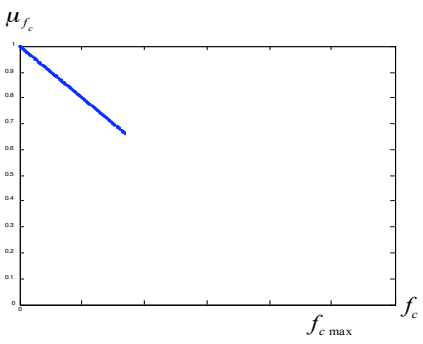

Fig. 1. Fuzzy membership function of the cost, $f_{c}$

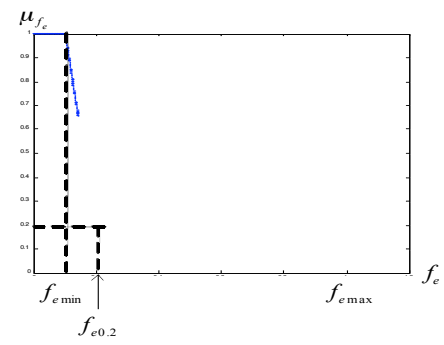

Fig. 2. Fuzzy membership function of power loss

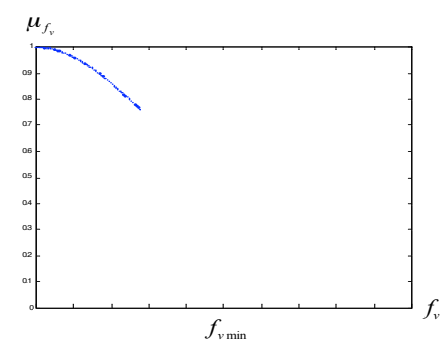

Fig. 3. Fuzzy membership function of the deviation of the bus voltage

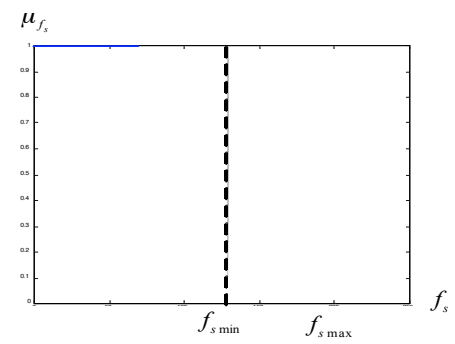

Fig. 4. Fuzzy membership function of the security margin of feeders and transformers

\subsection{Maximizing the Security Margin of Feeders and Transformers}

A simple index to assess the system security is the capacity margin of feeders and transformers.

$\min f_{s}=1-\min _{i}\left|\frac{I^{2}{ }_{\text {iRate }}-I^{2}{ }_{\text {iLoad }}}{I^{2}{ }_{\text {iRate }}}\right|, i=1,2, \ldots, n_{h}$

where $I_{\text {iLoad }}$ and $I_{\text {iRetad }}$ are the current flow and the rate 
flow of branch (transformer) $i$, respectively; $n_{h}$ represents the total number of branches (transformers), and $f_{s}$ denotes the security and system capacity index of the feeders. Lower $f_{s}$ implies more secure system capacity. Figure 4 plots the fuzzy membership function of the feeders (transformers).

\section{Immune Algorithm}

The immune system has a fundamental ability to produce new types of antibody or find the best-fitting antibody to attack an invading antigen [8, 9]. The immune system produces very many antibodies against innumerable, unknown antigen, by trial and error. The diversity of the immune system can be mathematically formulated as a multi-objective function optimization problem, with multiple solutions rather than single solution, to elucidate the diversity of antibodies that is essential to adaptability against foreign viruses and bacteria in the environment. A measure of diversity of antibodies produced from a lymphocyte population is required and must be defined. According to information theory, the entropy $H_{j}(N)$ of the jth gene is defined as $[8,9]$

$$
H_{j}(N)=-\sum_{i=1}^{N} p_{i, j} \log p_{i, j}
$$

where $p_{i, j}$ represents the probability that locus $\mathrm{j}$ is allele $i$. If all alleles at the jth gene are the same, then the entropy of the jth gene equals zero. The mean of the informative entropy in a lymphocyte population is represented by

$$
H(N)=\frac{1}{L} \sum_{j=1}^{L} H_{j}(N)
$$

where $H(N)$ denotes the mean of the informative entropy for all antibodies and $L$ is the size of the genes in an antibody. This entropy specifies the diversity of the lymphocyte population. Two expressions for affinity are considered in the presented approach. One $(A b)_{v w}$, is used to determine the diversity between two antibody $v$ and $w$ and can be represented as,

$$
(A b)_{v w}=\frac{1}{1+H(2)}
$$

where $H(2)$ quantifies the diversity between two antibodies, according to Eq. (7) for $N=2$. For $H(2)=0$, the genes of the two antibodies are identical. The other affinity $(A g)_{i}$ is that between antigen $A_{g}$ and antibody $A_{b}$ and is defined by

$$
\left(A_{g}\right)_{i}=\mu_{f_{i}}\left(A_{b i}\right)-\sum_{j=1}^{N_{c}} \mu_{g_{j}}\left(A_{b i}\right) \quad i=1,2, \cdots, N_{o}
$$

where $\mu_{f_{i}}\left(A_{b i}\right)$ is the value of the membership function for antibody $A_{b i}$ on objective $i ; \sum_{j=1}^{N_{c}} \mu_{g_{j}}\left(A_{b i}\right)$ are the values of the membership function with all applied constraints for antibody $A_{b i}$, and $N_{c}$ and $N_{o}$ are the numbers of constraints and objectives, respectively. The antibody is perfectly matched with the antigen when the affinity $(A g)_{i}$ equals one. Antibodies that have high affinities toward an antigen are selected to proliferate, while antibodies with low concentrations are suppressed. The concentration $c_{v}$ of each antibody can be defined as

$$
c_{v}=\frac{1}{N_{o}} \sum_{w=1}^{N_{o}} a c_{v, w}
$$

and

$$
a c_{v, w}= \begin{cases}1 & (A g)_{i} \geq \varepsilon \\ 0 & \text { otherwise }\end{cases}
$$

where $\varepsilon$ is a preset threshold. If $c_{v}\left(v=1,2, \ldots, N_{o}\right)$ is greater than a given threshold $\delta_{c}$, then this antibody becomes a memory antibody; else, it is suppressed. The goal of this step is to eliminate surplus solution candidates.

From the schema of the natural immune system, the mathematical optimization framework can be modeled as an algorithm, realized by the following steps.

Notably, in the above immune algorithm, the number of generated antibodies and the number of iterations can be experimentally determined. The rate of the crossover and mutation are also determined on a trial basis.

This study presents a two-stage immune algorithm embedded the compromise program to solve multiobjective problems.

In the first stage, the multi-objective optimization problem is transformed to a single objective optimization problem by selecting the kth objective as the primary objective function in turns $k=1,2, \ldots, N_{o}$ and converting the other objectives to constraints with individual maximum allowable values $\widehat{f}_{i}$ where $i=$ $1,2, \ldots, N_{o}$ and $i \neq k$.

In the second stage, the non-inferior set for all objectives is obtained by compromise programming. Compromise programming finds the best compromise with respect to all the objectives by computing a normalized Euclidean distance measure.

$$
D=\sum_{i=1}^{N_{o}} \frac{f_{i}(x)-\breve{f}_{i}}{\widetilde{f}_{i}-\breve{f}_{i}}
$$

This normalized Euclidean distance is used to evaluate how close the computed non-inferior solution is to the Pareto front. A smaller $D$ indicates the current computed non-inferior solution is closer to the Pareto front. For a multi-objective problem, the ideal value of each objective $\breve{f}_{i}$ (from stage 1 ) and the maximum allowable value of each individual objective $\bar{f}_{k}$ where $i$ and $k=1,2, \ldots, N_{o}$, can be used to express the overall 
multi-objective minimizing objective function, as follows.

$$
\operatorname{Min} D=\sum_{i=1}^{N_{o}} \frac{f_{i}(x)-\breve{f}_{i}}{\widehat{f}_{i}-\breve{f}_{i}}
$$

\section{Simulation Results}

The testing system includes seven branches and 69 buses, as presented in [3]. The presented method outputs five non-inferior solutions (options) with different features, one of which is to be selected by the decision-makers. Tables 1 and 2 compare the results with those in [3] and [10], in terms of the capacitor to be installed and power loss with and without compensation, respectively. In summary, the noninferior solutions obtained using the presented method, in terms of voltage deviation, power loss, cost and loading margin, are better than (or similar to) those obtained using the methods of [3] and [10].

TABLE I

CAPACITORS(kvar) TO BE INSTALLED

\begin{tabular}{c|r|r|r|r|r|r|r}
\hline & \multicolumn{7}{|c|}{ Methods } \\
\hline No. of bus & \multicolumn{5}{|c|}{ The proposed method } & Huang & Chiang \\
\hline 19 & 300 & 1200 & 300 & 600 & 300 & 600 & 300 \\
\hline 50 & 300 & 900 & 600 & 900 & 300 & 300 & 1200 \\
\hline 53 & 1200 & 600 & 600 & 600 & 600 & 300 & 0 \\
\hline Total_kvar & 1800 & 2700 & 1500 & 2100 & 1200 & 1200 & 1500 \\
\hline
\end{tabular}

TABLE II

THE RESULT OF THE REAL POWER LOSS (kw) WITH AND

\begin{tabular}{c|c|c|c|c|c|c|c|c}
\hline & \multicolumn{7}{|c|}{ WITHOUT INSTALLING CAPACITORS } \\
\hline $\begin{array}{c}\text { Load } \\
\text { level }\end{array}$ & $\begin{array}{c}\text { Without } \\
\text { compensation }\end{array}$ & \multicolumn{5}{|c}{ With compensation } \\
\cline { 3 - 9 } & & \multicolumn{5}{|c|}{ The proposed method } & Huang & Chiang \\
\hline Light & 538 & 393 & 457 & 347 & 401 & 337 & 347 & 345 \\
\hline Medium & 1,715 & 1,019 & 995 & 1,042 & 994 & 1,116 & 1,186 & 1,040 \\
\hline Peak & 3,190 & 1,865 & 1,752 & 1,965 & 1,806 & 2,134 & 2,276 & 1,964 \\
\hline Total_loss & 5,443 & 3,277 & 3,204 & 3,354 & 3,201 & 3,587 & 3,809 & 3,349 \\
\hline
\end{tabular}

\section{Conclusions}

Multi-objective optimization is of increasing importance in various fields, and has a diverse range of applications. Highly effective and efficient multiobjective algorithms can promote both scientific research and engineering applications in various areas. This work proposes the two-stage immune algorithm, embedding compromise programming, for solving the multi-objective capacitor placement problem. The concept of the non-inferior set is applied herein to obtain the set of optimal compromise solutions from which the decision maker can choose one. The simulation results indicate that the advantage of using the proposed technique is that it can find the best compromised solutions in a single run.

\section{References}

[1] M.E. Baran, and F.F. Wu, "Optimum Capacitor Placement on Radial Distribution Systems", IEEE Trans. Power Delivery, Vol. 4, No. 1, pp. 725-734, Jan. 1989.

[2] C.T. Su, and C.C. Tsai, "A New Fuzzy Reasoning Approach to Optimum Capacitor Allocation for Primary Distribution systems," in Proceedings of 1996 IEEE on Industrial Technology Conference, 1996, pp.237-241.

[3] H.D. Chiang, J.C. Wang, O. Cockings, and H.D. Shin, "Optimal Capacitor Placements in Distribution Systems: Part I: A New Formulation and Overall Problem", IEEE Trans. Power Delivery, Vol. 5, No. 2, pp. 634-642, April 1990.

[4] H.D. Chiang, J.C. Wang, O. Cockings, and H.D. Shin, "Optimal Capacitor Placements in Distribution Systems: Part II: Solution Algorithm and Numerical Results", IEEE Trans. Power Delivery, Vol. 5, No. 2, pp. 643-649, April 1990.

[5] Y.C. Huang, H.T. Yang, and C.L. Huang, "Solving the Capacitor Placement Problem in a Radial Distribution System Using Tabu Search Approach," IEEE Trans. Power Systems, Vol. 11, No. 4, pp.18681873, Nov. 1996.

[6] S. Sundhararajan, and A. Pahwa, "Optimal Selection of Capacitors for Radial Distribution Systems Using a Genetic Algorithm," IEEE Trans. Power Systems, Vol. 9, No. 3, pp. 1499-1507, Aug. 1994.

[7] F.C. Schweppe, H.M. Merrill, and W.J. Burke, "Least-cost Planning: Issues and Methods," Proceedings of the IEEE, Vol. 77, No. 6, pp. 899-907, June 1989.

[8] D. Dasgupta, Artificial Immune Systems and Their Applications, Springer-Verlag Berlin Heidelberg, Saladruck, Berlin, 1999.

[9] Y. Tsujimura and M. Gen, "Entropy-based Genetic Algorithm for Solving TSP," in 1998 Second International Conference on KnowledgeBased Intelligent Electronic Systems, No. 2, 2123 April 1998, Adelaide, Australia, pp. 285-290.

[10] S.J. Huang, "An Immune-Based Optimization Method to Capacitor Placement in a Radial Distribution System," IEEE Trans. Power Delivery, Vol. 15, No. 2, pp. 744-749, April 2000. 